\title{
Twenty Weeks Pregnancy with Giant Cyst: Anesthesia Problem and Perioperative Preparation
}

\author{
Diana Ch. Lalenoh, MD, Phd ${ }^{1}$ \\ Department of Anaesthesiology and Critical Care, Division of Neuroanesthesiology and Critical Care, Division of \\ Obstetric Anesthesia, Medical Faculty of Sam Ratulangi University \\ Prof. RD. Kandou Hospital/ Permata Bunda Hospital, Manado, Indonesia
}

\begin{abstract}
:
Background: Management of anesthesia in patients with ovarian cyst permagna is challenging because it is require careful preparation and have a high risk during the perioperative period. We report anesthetic management perioperatively in a woman,31 years, Gravid 5Para 2Abortus $2\left(\mathrm{G}_{5} \mathrm{P}_{2} \mathrm{~A}_{2}\right)$ with 19-20 weeks of pregnancy with ovarian cyst permagna and slight complaints of abdominal bloating,plan to elective surgery. We highlight the recent guideline for non-obstetric surgery in pregnancy

Objective: To report management of anesthesia in a woman with cystoma ovarian permagna

Case: A woman 31 years old attending with a growing abdomen experienced since the 19-20 weeks before admission. The patient complained of slight difficulty breathing. Her activity was mild decrease, with a slight difficult to walk.Abdominal circumference is $122 \mathrm{~cm}$. Ultrasound exmination showed a large cystoma with ascites. CT scan of the abdomen showed a large cyst mass with the size of $30.5 \times 33 \times 55.6 \mathrm{~cm}$ with solid components are urging the intestine and minimal intraperitoneal ascites fluid

Results: The surgery lasted for 2 hours and 30 minutes, cystoma successfully removed intact ovary weighed 23 $\mathrm{kg}$. Intraoperative bleeding patients about $800 \mathrm{ml}$, and $1000 \mathrm{ml}$ of urine production. After 32 hours of observation in the intensive care unit, patient was stable haemodynamic parameter, and allowed to be transferred in the ward
\end{abstract}

\section{BACKGROUND}

The presence of disease during pregnancy leading to alteration in the normal function of other system. It is important to treat the disease depending upon the severity and type of urgency. Several disease in pregnancy and management have been reported earlier but it is necessary to report a rare pathology, treatment option and its anesthetic management. ${ }^{1}$

Anesthetic management in pregnance woman with permagna ovarii cyst have big challenge because of high risk for both mother and the fetus, and so as perioperatively preparation. ${ }^{2,3}$ Large intra-abdominal tumours change the anatomy, physiology, and psychology of the patient. The musculature of the abdominal wall becomes stretched beyond the limits of rapid recovery as the tumour increases in size, and the patient may be rendered immobile. Pregnancy, a fibroid uterus, ovarian cyst, hydatid cysts, hydronephrosis, lymphomas, and fluid collections ay produce such swellings. Whatever the cause, the consequences of surgery are similar and attributable to the size of the tumour rather than to its distinctive pathology. It is with these problems that this article is concerned. ${ }^{4}$

The finding of an incidental adnexal mass in pregnancy has become more common with the routine use of ultrasonography in prenatal care. However, the management of asymptomatic adnexal masses that persist during pregnancy remains controversial. Although several institutions have published reviews of small numbers of cases of adnexal masses in pregnancy, no specific protocols exist for the appropriate management of these patients. In addition, no prospective studies randomising between observation and surgery have been performed. ${ }^{5,6}$

During pregnancy, surgery for non-obstetric procedures occurs in up to $2 \%$ of women. This figure may be considerably higher in the first trimester as pregnancy may go undetected at the time of surgery. Approximately $42 \%$ of procedures occur in the first trimester, $35 \%$ during the second and $23 \%$ during the third. The range and incidence of proceudres are similar to the non-pregnant group of young women. Acute abdominal problems are most common, with appendicectomy ranking first followed by cholecyctectomy. Pregnancy predisposes to cholelithiasis

${ }^{1}$ Corresponding Author: diana.lalenoh@yahoo.com 
and approximately 3\% of pregnant women develop gallstones, however only a limited number require surgery. Other common problems include adnexal disease (e.g. ovarian cysts which may rupture or become torted) and trauma. Much less common are cardiovascular or neurological emergencies. The demands of pregnancy on the cardiovascular system can cause decompensation of valvular heart disease or precipipitate aortic dissection. Anaesthetists who care for pregnant patients undergoing non-obstetric surgery must provide safe anaesthesia for both the mother and the foetes. To maintain maternal safety the physiological and anatomical changes of pregnancy must be considered and anaesthetic techniques and drug administration modified accordingly. Foetal wellbeing is related to avoidance of foetal asphyxia, teratogenic drugs and preterm labour. ${ }^{7}$

\section{CASe Report}

A 24-years-old parturient $\left(\mathrm{G}_{5} \mathrm{~A}_{2} \mathrm{P}_{2}\right)$ at 19-20 weeks of gestation presented at emergency department with. Patient was consulted to anesthetist by obstetric for elective caesarean section surgery because of. A lower segment caesarean section (LSCS) was plannned by the obstetric team and anaesthesia consultation was sought for it.

Her blood pressure was 130/80 $\mathrm{mmHg}$ Her pulse was 98 times/minute, respiratory rate was 28 times/minute, body temperature was $37^{\circ} \mathrm{C}$. Body weight was $60 \mathrm{kgs}$, and body height was $153 \mathrm{~cm}$. Haemoglobin $12.5 \mathrm{~g} / \mathrm{dL}$, haematocryte $38.7 \%$, thrombocyte $178,000 / \mathrm{mm}^{3}$, leucocyte $10100 / \mu \mathrm{L}$. Electrolyte (Na $135, \mathrm{Eg} / \mathrm{L}, \mathrm{K} 3.64 \mathrm{mEq} / \mathrm{L}$, Ca $98.7 \mathrm{mEq} / \mathrm{dL}$ )were normal limit.Electrocardiogram: Heart rate was 95 times/minute, with occasional ectopic beat (premature ventricular contraction/PVC) suspicious because of her cyst during pregnancy.By ultrasound, the fetus condition is in normal limit. We assessed the patient with physical status ASA III and then plannned to spinal anesthesia.

Blood products were kept available before surgery. She was transported to the operating theatre with nasal canule oxygen $2 \mathrm{~L} /$ minute. The problem detection in this patient were:. We give 2 lines of intravenous canules, in right arms and left arms, to kept IV drug emergency if needed. Premedication included IV ranitidine $(100 \mathrm{mg})$ and ondansetron $8 \mathrm{mg}$. O2, spinal anesthesia was achieved with $\mathrm{mg}$, fentanyl $25 \mu \mathrm{g}$, clonidine $75 \mu \mathrm{g}$. We give $2.5 \mathrm{mg}$ of midazolam during surgery as anxyolitic. During surgery blood pressure range between $100-120 \mathrm{mmHg}$, heart rate range between 60-74 times/minute. The operation performed in 2 hours and 15 minutes. The patient received $2000 \mathrm{ml}$ of crystalloids, and $500 \mathrm{ml}$ of colloids. Total bleeding during surgery was $750 \mathrm{ml}$. Total urin output during 2 hours and 15 minutes were $100 \mathrm{cc}$.Paracetamol $1000 \mathrm{mg}$ Inj was given for postoperative analgesia.

Post operative patient was transferred to the ICU for 1 day, and then to the ward for 5 days. After surgery, fetal heart examination was in normal rate, and the fetus was in normal condition by ultrasound examination.

\section{DISCUSSION}

\section{Physiological/anatomical changes during pregnancy}

\subsection{Cardiovascular Changes}

Maternal cardiac output increases in pregnancy by $50 \%$ and peaks by the end of the 2 nd trimester. This due to a combination of an increased heart rate (25\%) and stroke volume (30\%). The increase in heart rate is a reflex response to a lowered systemic vascular resistance (SVR) caused by circulating oestrogen and progesterone. Left ventricular hypertrophy and dilatation facilitate the increase in stroke volume but myocardial contractility remains unchanged. Electrocardiogram changes that occur in pregnancy and are entirely normal include left axis deviation and minor ST/T wave changes. Heart murmurs are also common due to turbulance associated with increased blood flow. In this case, patients presenting with paroxysmal premature ventricular beat before surgery. In operative period, haemodynamic was stable, without any premature ventricular beat. This condition may be because of adequate analgesia, anxyolitic for the patient, besides adequate relaxation for surgery facilitating. ${ }^{7-9}$

As the enlarging uterus moves out of the pelvis it can compress the inferior vena cava and the descending arota in the supine position. The compression of the inferior vena cava causes decreased venous return and hence preload, which reduces cardiac output by up to $20 \%$. This is known as supine hypotension syndrome. Pregnant patients compensate for hypotension by an increase in sympathetic tone causing vasoconstriction and tachycardia. This may divert blood away from organs such as the uterus, with subsequent foetal distress. The compression of the aorta can cause a further reduction in uterine blood flow. Aortocaval compression becomes clinically relevant from approximately 20 weeks gestation. It can be relieved by a left lateral tilt of 15 degrees, which is therefore essential in all pregnant patients in the supine position after 20 weeks. This is especially important to remember when a patient is under regional anaesthesia/analgesia since hypotension may be potentiated by a sympathetic block. There is an 


\section{American Research Journal of Medicine and Surgery, Volume 1, Issue 2, 2015}

ISSN 2379-8955

increase in blood volume in pregnancy of between $35-50 \%$ at term. There is both an increase in plasma volume and red cell volume, but a greater increase in plasma volume which leads to a dilutional anaemia. The reduced blood viscosity aids flow through the uteroplacental circulation and the increase in volume serves as a protective measure against haemorrhage at delivery. It must be remembered that because of the increase in blood volume, along with a resting tachycardia, there may be delay in the onset of the classical symptoms and signs of hypovolaemia. Pregnancy is a hypercoaguable state with an increase in most clotting factors. The platelet count may fall but there is actually an incerase in production and consumption. Pregnancy is a significant risk factor for thromboembolism and therefore thromboprophylaxis is essential in the postoperative period when the risk is further increased by immobility and the hypercatabolic state. We must remember left lateral tilt to prevent aortocaval compression, remember meticulous pre-oxygenation to prevent hypoxia, remember antacid prophylaxis and rapid sequence induction to reduce risk of aspiration. . $^{10,11}$

\subsection{Respiratory Changes}

The respiratory changes of pregnancy are perhaps the most important for anaesthetists to note. There is an increased oxygen demand of up to $60 \%$ at term. This is met by an increased cardiac output and an increase in minute ventilation (MV). Minute volume increases early due to an increase in respiratory rate and tidal volume and is up by $45 \%$ by term. This increase in MV is mediated by progesterone which acts as a respiratory stimulant. The increased MV causes a mild respiratory alkalosis $(\mathrm{PaCO} 2$ decreases by $1 \mathrm{kPa}) .^{7-9}$

The increase in $\mathrm{pH}$ is limited by increased renal bicarbonate excretion. Relative hypocapnia should be maintained when artificially ventilating pregnant patients. An increase in maternal PaCO2 limits the gradient for $\mathrm{CO} 2$ diffusion from foetal to maternal blood leading to foetal acidosis. The functional residual capacity (FRC) is the maternal blood leading to foetal acidosis. The functional residual capacity is the main oxygen reserve in the apnoeic patient and is decreased in pregnancy due to enlarging uterus displacing the diaphragm upwards. This is further exacerbated in the supine position and increases as the pregnancy progresses. ${ }^{7,9}$

Airway management may be challenging during pregnancy. Bag-mask ventilation may be more difficult due to increased soft tissue in the neck. Laryngoscopy can be hindered by weight gain and breast engorgement. Increased oedema of the vocal cords due to increased capillary permeability can hinder intubation and increase the risk of bleeding. This may make further attempts at intubation more difficult and increase the incidence of failed intubation. Increased maternal oxygen consumption and reduced FRC results in rapid oxygen desaturation during attempts at intubation. Smaller sized endotracheal tubes may be needed and all anaesthetists should be familiar with a failed intubation drill. Nasal intubation should be avoided due to increased vascularity of mucous membranes. Given the combination of these changes, careful pre-oxygenation is essential prior to induction of anaesthesia. This should be confirmed if possible by monitoring the end tidal oxygen fraction. In a well pre-oxygenated patient this should be > 0.9. Pre-oxygenation can be less efficient in the term parturient in the supine position because the closing volume of the alveoli may be greater than the FRC. Pre-oxygenation in a slightly head up position may help this. ${ }^{11,12}$

In order that, we choose regional anesthesia for this patient, to kept adequate respiratory and better airway management. Because regional anesthesia is less impact in FRC and MV.

\subsection{Gastrointestinal Changes}

Circulating progesterone reduces the lower oesophageal sphincter (LOS) tone, increasing the incidence of oesophageal reflux. This is further exacerbated by anatomical changes. The gravid uterus is displaced upwards and to the left pushing the intra-abdominal part of the oesophagus into the thorax in most pregnant women. This often makes the LOS incompetent and lowers the barrier pressure. These factors, along with a lowered stomach $\mathrm{pH}$, invrease the risk and severity of aspiration pneumonitis under general anaesthesia. ${ }^{11-13}$

It is recommended that from 16 weeks gestation, patients undergoing general anaesthesia should be given prophylaxis against aspiration pneumonitis. This usually includes a non-particulate antacid such as sodium citrate $.3 \mathrm{M} 30 \mathrm{ml}$ and $\mathrm{H} 2$ receptor antagonist e.g. ranitidine $150 \mathrm{mg}$ orally or $50 \mathrm{mg}$ intravenously. Some anaesthetists may also choose to give a prokinetic such as metoclopramide. Induction of anaesthesia should be by a rapid sequence technique which cricoid pressure and a fast acting muscle relaxant such as suxamethonium. A cuffed endotracheal tube should be used. At the end of the procedure patients should be extubated fully awake in the lateral position. ${ }^{7}$

\subsection{Drugs and Teratogenicity}

Teratogenicity is defined as the observation of any significant change in the function or form of a child secondary to prenatal treatment. The teratogenicity of a drug depends uopn the dose administered, the route of administration, the 
timing of foetal exposure and the species administered to. During the first two weeks of human gestation the teratogens have an all or none phenomenon; the foetus is lost or is preserved fully intact. The period from the 3rd to the 8th week of gestation, represents the most important time for organogenesis during which drugs can exert their most serious teratogenic effects.

After this, drug exposure should not cause organ abnormalities, but foetal growth retardation may occur. Although most anaesthetic drugs are known teratogens in certain species most agernts are safe in humans. The foetus is at more risk from asphyxia than the teratogenic effect of anaesthetic drugs. Studies looking at the outcomes of women who underwent surgery during pregnancy suggest no increase in congenital anomalies in their offspring but an increase in foetal loss, growth restriction and low birth weight attributed to the requirement for surgery (not anaesthetic administration). There is some concern from animal and epidemiological studies that exposure to general anaesthetic agents may cause neurodevelopmental delay in infants. It is difficult to extrapolate animal findings to humans and in epidemiological studies it is difficult to distinguish the potential confounding effects of anaesthesia, reason for surgery and underlying medical conditions.

Nitrous oxide inhibits methionine synthetase, and therefore there is concern it could affect DNA synthesis in the developing foetus. It has also been shown to be teratogenic during peak organogenesis in rodents, but there is no evidence in humans. Anaesthesia can be safely delivered without nitrous oxide and therefore many would avoid its use during non-obstetric surgery in the pregnan woman. Another drug of concern is ketamine. This causes increased uterine tone and foetal asphyxia and should not be used in the first two trimesters. The effect is not seen in the third trimester. Benzodiazepines have been associated with a cleft lip and palate in animal studies. Benzodiazepines have been associated with a cleft lip and palate in animal studies. The association in humans is controversial. A single dose has not been associated with teratogenicity. Long term use should be avoided as neonatal withdrawal may occur. Single doses may be useful to prive anxiolysis preoperatively. ${ }^{7}$

In this case, we discuss with surgeon and the surgeon agree to make laparatomy with the incisional procedure below the umbilicus. We decide to perform spinal anaesthesia with bupivacaine $0.5 \% 20 \mathrm{mg}$ added with fentanyl 25 and clonidine $75 \mu \mathrm{g}$. This adding agents performed to longer blockade period. If the surgeon performed laparascopy, we must change the technique with general anaesthesia because of $\mathrm{CO} 2$ gases that they used during laparoscopy. But general anaesthesia too risky in this patients.

\subsection{Laparoscopic Surgery}

There were previous concerns regarding foetal safety during laparoscopic surgery. These included fears of direct uterine and foetal trauma, foetal acidosis due to absorbed carbon dixoide and decreased maternal cardiac output secondary to the increased intra-abdominal pressure and positioning with a subsequent decrease in uteroplacental perfusion.

There are advantages to laparoscopic surgery for both the mother and foetus such as decreased post-operative pain (and therefore less need for analgesiscs), shorter recovery times and a lower risk of thromboembolic events. A Swedish study compared laparotomy and laparoscopy performed in pregnancy in over 2 million deliveries. Premature delivery, growth restriction and low birth weight were more common in both groups compared to the general population but there were no differences between the laparotomy and laparoscopy groups.

Pregnancy should therefore not be seen as a contraindication to laparoscopic surgery if surgery is required. Certain precautions should be taken. Pneumatic stockings should be used to promote venous return and the lowest pressure pneumoperitoneum $(<12 \mathrm{mmHg}$ ) should be used where possible. Aortocaval compression should be avoided and changes in position should be undertaken slowly. $\mathrm{PaCO} 2$ should be closely monitored by the routine use of end tidal carbon dioxide monitoring and consideration of arterial blood gas analysis in selected cases. Foetal heart rate monitoring may be advisable to detect foetal compromise early allowing optimisation of maternal haemodynamic. Foetal heart rate changes may indicate the need for temporary deflation of the pneumoperitoneum

\section{MANAGEMENT}

Cyst less than 6 centimetres in diameter and appearing benign on ultrasound are generally treated conservatively as they may undergo spontaneous resolution. Corpus lueal cysts regress by 12 to 16 weeks. Cysts more than 10 centimmetres in size are usually resected due to increased risk of malignancy, rupture or torsion. Management of cysts between 5 to 10 centimetres is controversial. If the cysts contain septae, nodules, papillary excrescences or solid components then resection is recommended. Those with simple cystic appearance may be managed expectantly with serial ultrasound surveillance. However the may require emergency exploratory laparotomy for rupture, torsion 
or infarction in as many as 50\% cases. With the advent of imaging techniques like high resolution ultrasound MRI and transvaginal colour Doppler, the expectant management has become much more common. If the ovarian cyst is diagnosed in the first trimester, it is better to wait till 16 meeks whenn the implantation of pregnancy is more secure and also the cyst may dissapear spontaneously. So as in this case, surgeon decide to operate this patient in 19 weeks of gestation.

Persisting tumours are treated by cystectomy or ovariotomy as indicated. Ovarian tumour or cyst can be easly removed till 28 weeks of gestation thereafter it is not readily accessible and may precipitate preterm labour. Ovarian cyst which rupotures, or undergoes torsion or of it shows evidence of malignancy, requires immediate surgery, irrespective of the period of gestation. A simple cystectomy can be performed in the abscence of overt malignancy. Previously untwisting of the pedicle was avoided to prevent emboli and toxic substances related to hypoxia, from entering peripheral circulation. Recently, re-establishing ovarian circulation by untwisting, has shown to result in viable ovarian tissue with no systemic complication.

Ovarian torsion is relatively uncommon in the second trimester of pregnancy. Diagnosis can usually be made on the basis of the characteristic clinical presentation in conjunction with ultrasound (US) evidence of a unilaterally enlarged adnexal mass. Treatment options are limited to surgery, either by laparoscopy or laparotomy, but the former becomes more difficult after second trimester. ${ }^{9}$

\section{SURGERY DECIDED}

Surgery is indicated when physical examination or imaging of a pregnant woman reveals in adnexal mass that is suspicious for malignancy, but the physician must weigh the benefit of prompt surgery against the risk to the pregnancy. This wquation can be complicated in several ways. For example, surgical staging of clinically early ovarian cancers is more difficult due to the pregnanc uterus, which is more extensively manipulated during these procedures. In addition, an optimal operation sometimes necessitates removal of the uterus.

\subsection{When The Problem Is Acute}

In rare cases, a pregnant patient will have (or develop during observation) an acute problem due to torsion or rupture of an adnexal mass. Some ovarian cancers may present acutely, such a rapidly growing malignant germ-cell tumor or a ruptured and hemorrhaging granulosa-cell tumor. Emergent surgery is necessary to manage the acute adnexal disease and reduce the likelihood of pregnancy loss. These events are infrequent, occufing in less than $10 \%$ of women with a known, persistent adnexal mass during pregnancy. Furthermore, recent studies have not found a substantial pregnancy complication rate associated with such emergency surgeries. ${ }^{10,11}$

\subsection{Fast Track Adnexal Masses In Pregnancy}

Elective surgery for an adnexal mass anytime during pregnancy increases the risk of pregnancy loss and the likelihood of intrauterine growth restriction (IUGR) and preterm delivery. A 1989 study from Sweden defined a cohort of 5,405 women (from 720,000 births) who were known to have a nonobstetric operation while pregnant, with the following results: Congenital malformation and stillbirth were not increased in the weomen undergoing surgery; the number of very-low-and lowbirth-weights infants did rise, however-the result of both prematurity and IUGR; also elevated was the incidence of infants born alive but dying within 168 hours; these risks increased regardless of trimester; No specific type of anesthesia or operation was associated with adverse reproductive outcomes, and the cause of those adverse outcomes was not determined. Some recent data suggest that adnexal surgery during the late second or early third trimester poses the greatest risk of preterm delivery or IUGR or both.

\section{WINDOW OF OPPORTUNITY: EARLY TO MID-SECOND TRIMESTER}

During this time frame, elective surgery for an adnexal mass still affords some pelvic exposure without the need for significant uterine manipulation and has been associated with a lower risk of pregnancy complications. The other window for operation is at the time of cesarean section. An elective cesarean section is sometimes performed specifically to manage a persistent adnexal mass. Among the factors that warrant consideration when contemplating this approach are the elective uterine incision (with its attendant implications for future pregnancies), the higher risks associated with cesarean delivery in general, the type of skin incision (a vertical incision is appropriate in the event of ovarian malignancy). The potential for better exposure or laparoscopy at a later date, the increased difficulty of ovarian cystectomy at the time of cesarean section, and the patient's wishes. 


\section{LAPAROSCOPY OR LAPAROTOMY TECHNIQUE}

The dataon laparoscopy during the first and second trimesters of pregnancy indicate that it is as safe as laparotomy. A 1997 Swedish study identified cohorts of 2,181 women undergoing laparoscopy and 1,522 women undergoing laparotomy (from a total of 2,015,000 deliveries) between the fourth and 20th weeks of pregnancy. In both groups there was an increased risk for the infant to weigh less than 2,500 g, to be delivered before 37 weeks, and to have IUGR. There were no differenced between the 2 groups for these of 2,181 women undergoing laparoscopy and other adverse outcomes. Small series of laparoscopic procedures to manage an adnexal mass during pregnancy suggest that this approach is most applicable during the first (for highly selected emergent cases) or early second trimester to manage masses less than $10 \mathrm{~cm}$ in diameter, particularly when adnexectomy is planned.

Laparoscopy may be considered "minimmally invasive" because it reduces manipulation of the pregnant uterus during adnexal surgery. However, it is more difficult to assess and remove ovarian cysts laparoscopically, althoufh an early ovarian malignancy could be staged via laparoscopy by an experienced surgeron.

\subsection{Considerations during laparotomy}

When performing a laparotomy or cesarean section for an adnexal mass, the surgeon must take into account a number of variables when selecting the type of incision (ie, vertical vs transverse). In general, if malignancy is suspected, or if uterine manipulation is to be minimized, a vertical incision is the best. Other considerations include a prior scal, body habitus, obstetric issues, and the patient's wishes. ${ }^{10,12}$ Ovarian cysts over 5 and $15 \mathrm{~cm}$ in diameter are described as large and giant, respectively. In addition, women having large cysts without regression in 6-8 weeks time are candidates for surgery. Although data has been published on laparoscopic or laparoscopy assisted menagement of large and giant cysts, midline laparotomy is still preferred by many surgeons, particularly in cases of giant cycsts. ${ }^{13}$

Laparoscopic approach is more advantageous over laparotomy, considering better cosmetic results, lesser blood loss, lesser pain and analgesic requirement, faster recovery, and shorter hospitalization time. Laparoscopic approach to giant ovarian cyst, in cases when the cysts sizes exceed to the umbilicus, may be difficult regarding the risk of cyst rupture and limited working space. However, if the laparotomy is choosen as the operative treatment, a larger incision is required to excise the cyst. Kim et al found that only $0.2 \%$ incidence of chemical peritonitis following laparoscopic removal of dermoid cysts. ${ }^{14}$

Laparoscopic approach allows proper exposure of $\mathrm{Cul} \mathrm{de} \mathrm{sac} \mathrm{and} \mathrm{allow} \mathrm{forceful} \mathrm{jet} \mathrm{lavage} \mathrm{aspiration} \mathrm{ensuring} \mathrm{pelvic}$ clean out from any microscopic material of the dermoid cyst. A situation may not be available during open laparotomy. ${ }^{15}$

\subsection{Laparotomy}

A laparotomy is a surgical procedure through an opening (that is larger than that used for laparoscopy) into the abdomen. A laparotomy is normally used when a laparoscopy cannot be done effectively. Some reasons for laparotomy are removal of ovarian cysts, removal of an ovary, or removal of fibroids and/or adhesions.

The risks of developing a problem that is related to having a laparotomy is low compared to the other major surgery. However, problems can occur. Problems that could develop include: 1) A bad reaction to anesthetic. This is minimal in an otherwise healthy person. 2) Blood clots in the deep veins of the legs or lungs. These can be prevented by early ambulation and the short term use of anti-coagulation medication. 3) Infection. This can be successfully treated with antibiotics. 4) Bleeding. There will be a small amount of blood loss during surgery. In rare cases, if there is a large amount of blood lost, a blood transfusion is required. 5) Injury to the nearby organs such as the urinary tract or bowel. If these injuries do occur, they are usually addressed at the time of the surgery without any significant longterm problems. ${ }^{16,17}$

So in this case we decide to laparotomy the patient because of the possibility of malignancy besides anesthetic important factor that can cause theratogenic if use some anesthetic drug in general anesthesia technique. With laparotomy procedure by surgeon, anesthetic management can performed by spinal anesthesia.

The common non-obstetric surgeries during pregnancy are ovarian cyst, appendictomy, Shirodker's operation etc. Regional anaesthesia is preferred as surgery whereas general anaesthesia is associated with higher incidence of abortions, due to uterine relaxant effect of volatile anaesthetic and compromised uteroplacental blood flow. Commonly used anaesthetic agents are not teratogenic. Theratogenic sodium, succinylcholine, nitrous oxide and halothane have no teratogenic effects. Induction of teratogenicity and induced abortions are more common in first 
trimester and premature labor in third trimester. Lesion caused by trauma/ tumor do not prevent conception and continuation of pregnancy. But pregnancy is likely to be complicated by urinary tract infection, anemia, pressure necrosis of skin and worsening of the disease itself. ${ }^{18}$

Surgery during pregnancy is relatively common. The present review of the literature will focus on relevant issues such as maternal safety during non-obstetric surgery in pregnancy, teratogenicity of anesthetic drugs, the avoidance of fetal asphyxia, the prevention of preterm labor, the safety of laparoscopy, the need to monitor the fetal heart rate and will finally give a practical approach to manage these patients. The most common indications for surgery during pregnancy are either pregnancy related or nonpregnancy related. Pregnancy related surgery includes interventions for cervical incompetence and surgery for ovarian cyst problems. Increasingly popular is also fetal surgery with an estimated 250 to 500 cases performed now each year in the Europe. ${ }^{19,20}$

The most common non pregnancy related indications are acute abdominal problems (most commonly appendicitis and cholecystitis), maternal trauma, and surgery for maternal malignancies. Of course any type of emergent surgery may be carried out during pregnancy. Surgery may be indicated during any stage of pregnancy. In a Swedish registry of 5405 patients who had surgery during pregnancy, $42 \%$ had an intervention during the 1 st trimester, $35 \%$ during the 2nd trimester and 23\% during the 3rd trimester. When caring for pregnant women undergoing nonobstetric surgery, anesthetists must provide safe anesthesia for both mother and child. Maternal safety is related to the physiologic adaptations associated with pregnancy, which enforce anesthetists to adapt their standard anesthetic techniques. Fetal safety relates to teratogenicity, avoidance of fetal asphyxia and avoidance of preterm labor and delivery. ${ }^{18-20}$

\section{SPINAL ANESTHESIA}

Spinal anesthesia is a generally preferred anesthetic technique as it is simple to perform; it provides rapid onset and a dense block. It also provides excellent post-operative analgesia when intrathecal opioids are used. It has no effect on Apgar scores and umibilical artery $\mathrm{pH}$ in preeclampsia as long as the systolic blood pressure is maintained greater than $80 \%$ or more of the baseline. The incidence of spinal induced hypotension and the vasopressor requirement were found to be two times lower in preeclamptic parturients when compared with normal parturients undergoing CS delivery. The increased production of circulating factors with potent pressor effect and the increased sensitivity to vasopressor drugs in preeclampsia along with the use of hyperbaric bupivacaine (8-12 $\mathrm{mg}$ ) with opioids could decrease the spinal induced hypotension in preeclamptic parturients. Cardiac output monitoring after spinal anesthesia has shown that neither spinal anesthesia nor the use of phenylephrine to treat hypotension reduce cardiac output during CS delivery, further supporting its safety in preeclamptic parturients. ${ }^{18-20}$

Continuous spinal anesthesia offers the flexibility of titration of local anesthetic agents in small aliquots; thus, graded sympathetic block could be achieved with a lower degree of sympathectomy in these parturients. However, the higher rate of infection, injury to nerve roots, post dural puncture headache and technical difficulty are potential pitfalls and this technique is not frequently used. ${ }^{18,19}$

\section{EPIDURAL ANESTHESIA}

Epidural anesthesia may be instituted in a step like fashion for non-emergency CS delivery with an infusion of 500$1000 \mathrm{ml}$ intravenous crystalloid that will sufficiently minimize maternal hypotension just as with non-preeclamptic patients. Van de Velden et al use administer incremental dose of $0.5 \%$ bupivacaine $(3 \mathrm{ml})$ in a dose sufficient to obtain a T8/ T10 dermatomal sensory level of analgesia with monitoring of maternal vital signs and fetal heart rate tracing. Once the epidural catheter has been proven functional with stable maternal hemodynamics and fetal heart rate tracing, surgical anesthesia may then be achieved to the T4 sensory dermatome necessary for cesarean delivery. In a prospective observational study involving 12 preeclamptic parturients undergoing elective cesarean delivery, preloading with crystalloids has not shown to decrease the incidence of maternal hypotension and changes in the uterine artery velocity waveforms were minimal when maternal systolic blood pressure were maintained $80 \%$ or more of baseline. Similarly, in a recent retrospective review comparing two tertiary referral obstetric units has shown that preeclamptic parturients with liberal fluid administration had a higher incidence of acute pulmonary edema over parturients with restricted fluid administration during the perioperative period. The use of fentanyl (< $2 \mathrm{mcg} / \mathrm{kg}$ ), a rapid and short-acting opioid, may increase the rapid onset and prolong the duration of anesthesia. The use of epinephrine-containing local anesthesia has not shown altered Apgar scores and or umbilical blood gases. ${ }^{19,20}$

\section{COMbined SPINAl EPIDURAL ANESTHESIA}

Combined spinal epidural (CSE) provides the reliability of spinal anesthesia and at the same time the flexibility of good postoperative pain relief through an epidural catheter infusion. The epidural component of the CSE offers superior hemodynamic stability over spinal anesthesia. There is no difference in neonatal outcome between spinal 
and epidural anesthetic techniques. A small prospective, randomized study involving 30 women with severe preeclampsia undergoing elective cesarean delivery, the CSE technique was found to be a safe alternative study involving 28 severe preeclamptic parturients undergeoing elective cesarean delivery, a dose of intrathecal bupivacaine $7.5 \mathrm{mg}$ with fentanyl $25 \mathrm{mcg}$ was found to maintain better hemodynamic stability over parturients who had $10 \mathrm{mg}$ of intrathecal bupivacaine with fentanyl $25 \mathrm{mcg}$ and it was equally effective for elective CS delivery. In a recent study involving 18 preeclamptic and normal parturients undergoing CS delivery, the ED50 of intrathecal bupivacaine was similar in both groups ( $4.7 \mathrm{mg}$ of bupivacaine $+20 \mu \mathrm{g}$ of fentanyl). Uneventful spinal anesthesia for elective cesarean delivery in severe preeclamptic parturients has been administered using bupivacaine in a dose range of 8-12.5 mg along with opioids including morphine $100 \mathrm{mcg}$, fentanyl $10-25 \mathrm{mcg}$ and sufentanil 3-5 mcg. ${ }^{19,20}$

\section{GENERAL ANESTHESIA}

When there is an immediate threat to the mother or fetus, GA with rapid sequence intubation may be considered over RA due to its rapid onset of anesthesia, control over the airway and potential for less hypotension than RA. However, a high degree of anticipation of difficult endotracheal intubation must be considered due to increased airway edema, short neck and large breasts that can obscure the laryngoscopic view during intubation. It is essential to keep the difficult airway cart available while planning GA. Similarly, with the pre-existing elevated blood pressure, techniques to attenuate the hemodynamic response to intubation with minimal effects on the fetus should be considered. The administration of magnesium sulfate pre-operatively could potentiate the duration of action and sensitivity of succynilcholine. Similarly, the uterine atony and coagulopathy from magnesium therapy may cause considerable intrapartum blood loss. Hence, a wide-bore intravenous access and blood products should be kept available prior to anesthesia. The triennium report from the Center for Maternal and Child Enquiries, reported two cases of direct anesthetic deaths on administration of GA to parturients due to failure to ventilate the lungs and aspiration of gastric contents in the post operative period. When there is a maternal or fetal compromise without any threat to either of them, the management depends on the availability of time to perform or activate RA. ${ }^{18-20}$

The clinical assessment of maternal and fetal status along with a clear communication with the obstetrical team may play a vital role in the decision of the anesthetic technique. With the availability of time and no threat to either mother or fetus, an epidural top up of a pre-existing epidural catheter with rapid acting LA or a subarachnoid block with low dose LA and opioid may be considered. On the contrary, when there is a threat to mother or fetus with insufficient time to perform a regional anesthetic technique, GA with peroperative precautions may be considered over regional anesthetic technique. ${ }^{19,20}$

Subarachnoid block with intrathecal opiod or CSE anesthetic technique may be considered over GA when there is no maternal or fetal compromise. The administration of neuraxial anesthesia minimizes the risk of neonatal exposure to potentially depressant anesthetic drugs, decreases the risk of maternal pulmonary aspiration, promotes early ambulation and reduces the incidence of maternal thromboembolism. Similarly, the administration of titrated opioids along with LA during neuraxial anesthetic techniques offers postoperative analgesia and minimizes the consumption of systemic opioids. ${ }^{19,20}$

Different non obstetric surgeries are performed during pregnancy, depending on the urgency and emergency. Surgery should be postponed if the disease doesn't require emergency intervention, in view of maternal and fetal well-being. The surgery was planned in our patient to treat it and also to prevent further complications. Main aim should be to save the life of mother while taking care to minimize the risk of miscarriage and preterm delivery. Aspiration prophylaxis injection ranitidine and injection of metoclopramide (IV) was given prior to surgery. It is important to consider the pregnant patient as full stomach and follow the guidelines. The most important and serious risk to the fetus is intrauterine asphyxia during maternal surgery. According to the latest guidelines by "The Americaln College of Obstetricians and Gynecologist", pre and post surgical procedure confirmation of fetal heart rate by Doppler is generally sufficient if the fetus is pre-viable. Intra-operative electronic fetal monitoring should be done if the fetus is viable, physically possible to perform, patient has given informed consent for emergency cesarean delivery, obstetrician is available and willing to intervene during the surgical procedure for fetal indications and if the nature of the planned surgery guidelines by The American College of Obstetricians allows the safe interruption or alteration of the procedure to provide access to perform emergency delivery. The left uterine displacement is recommended in pregnant patients of 20 weeks and above. ${ }^{17-19}$

A combination of general and epidural anesthesia reduces analgesic and anesthetic agent requirement, better intraoperative hemodynamic stability, suppressed metabolic, endocrine and immunologic responses. Management of these responses with good post-operative morbidity and mortality. Hence the combination has an advantage of early recovery and mobilization. The main controversy lies in type of anesthesia for non-obstetric surgery during pregnancy since all general anesthetic drugs cross the placenta. It is important that whatever type of anesthesia, the 
goal should be to maintain stable hemodynamic, perioperative good analgesia, avoid drugs having teratogenicity, maintain good utero-placental flow and prevent intraoperative fetal hypoxia and acidosis. Studies have shown that hypnotic drugs, opioids and sedatives do not have deleterious effects on the developing embryo or fetus. Since there is weak evidence that nitrous oxide leads pregnancy loss and vasoconstructive property it should be avoided. ${ }^{1}$

All anesthetic agents including volatile agents can be used in pregnancy. Volatile anesthetic agents prevent premature uterine activity. Many studies have shown regional anesthesia is better than general anesthesia due to two things, first, avoidance of poly-pharmacy and second avoidance of the airway. The largest risk of regional anesthesia is hypotension resulting from sympathetic nerve blockade there by reducing uterine blood flow and perfusion to the fetus. The anesthesia technique should be individualized depending upon surgical site. The general anesthesia is not an absolute contraindication but if some important precautions such as, prophylaxis against aspiration, avoidance of unnecessary drugs and proper airway management are followed, and then it can be a superior technique. Whenever possible epidural should be combined with general anesthesia due to various advantages as mentioned above. ${ }^{17,19,20}$

It is important to monitor and maintain in normal ETCO2 during general anesthesia, as hypocarbia due to hyperventilation impairs fetal oxygen delivery by shifting maternal oxygen-hemoglobin dissociation curve to the left. Fetal respiratory acidosis can be a result of maternal hypercarbia which can lead to fetal myocardial depression. Hypercapnia also results in uterine artery vasoconstriction and reduced uterine blood flow. ${ }^{18-20}$

Different tocolytics like calcium channel blockers, magnesium, beta agonists and oxytocin receptor blockers are used depending upon the pregnancy period to prevent preterm delivery. Post-operatively, patients should be monitored for contractions and treated with tocolytic agents when appropriate. It is important to keep in mind that these drugs may interact with anesthetic drug. Several obstetrician advised injection progesterone which was given peri-operatively. Animal studies showed that the progesterone at higher dosage decreases the minimum alveolar concentration value of sevoflurane..$^{17,18,20}$

If the treatment options are considered the minimal invasive or endoscopic surgeries have higher advantages than open surgery due to no or minimal exposure to anesthetic drugs, lesser surgical stress, avoidance of prolonged hospital stay, early recovery and lesser economic burden. A recent study showed cysto-gastrostomy when performed endoscopically has higher advantages than open surgical technique. It is important to know all types of treatment options are not available in developing and underdeveloped countries but it is always better to consider for minimal invasive procedure whenever possible. ${ }^{19,20}$

\section{Postoperative CARE}

As previously stated pregnancy induces a hypercoaguable state and the risk of thromboembolic disease is further increased by postoperative venous stasis. Attention to thromboprophylaxis is therefore essential. This should include early mobilization, maintaining adequate hydration, TED stockings and other calf compression devices and consideration of pharmacological prophylaxis (for example subcutanoeus low molecular weiht heparin).

\section{ANAlgeSia}

Adequate analgesia is important as pain will cause increased circulating catecholamines which will impair uteroplacental perfusion. Analgesia may mask the signs of early preterm labour and therefore tocometry is useful to detect contractions. This will enable tocolysis to be administered without delay. If a pregnancy continues beyond the first postoperative week the incidence of premature labour is no higher than the non-surgical pregnant patient.

The common analgesic drugs used are classified by the FDA and their safety in breastfeeding. Although laparotomy is still considered the standard for ovarian cyst removal, over the past 15 years minimally invasive surgery has gained wider acceptance in cases where preoperative assessments suggests an adnexal mass is benign. Unfortunately, minimally invasive management of a large ovarian cyst (greater than $10 \mathrm{~cm}$ ) is particularly challenging for several reasons: *) The cyst can rupture and spill its contents into the peritoneum; *) An unexpected malignancy may be revealed. An innovative minilaparotomy technique for the removal of benign ovarian cysts offers the advantages of laparoscopic and laparoscopic-assisted procedures while bypassing the major disadvantages: the necessity for specialized and expensive equipment, lengthy operative time, and long learning curves. (The minimally invasive procedures currently available for the treatment of ovarian cysts include laparoscopic cystectomy, laparoscopic-assisted minilaparotomy cystectomy, laparoscopic-assisted vaginal cystectomy, combined percutaneous ultrasound cyst aspiration and laparoscopic cystectomy, transvaginal cystectomy, and the traditional minilaparotomy cystectomy. ${ }^{8}$ The commonest type of ovarian tumours encountered in pregnancy are cystic teratoma, paraovarian cyst, serous cystadenoma, corpus luteal cysts, luteomas, etc. Serous cystadenomas are thin walled, translucent cysts usually unilocular, may have few daughter cysts, varying between 20-30 cms in size. They are often unilateral can be bilateral. 10-15\% of them are borderline malignant while 20-40\% 


\section{American Research Journal of Medicine And Surgery, Volume 1, Issue 2, 2015}

ISSN 2379-8955

are malignant. Differential diagnosis includes: uterine leiomyomas, non pregnant horn of bicornuate uterus, appendiceal abscess, diverticular abscess, pelvic kidney, retroperitoneal tumours, ectopic pregnancy and retroverted gravid uterus.

Complications of the cysts associated with pregnancy are torsion of the cyst, rupture, infection, malignancy, impaction of cyst in pelvis causing retention of uterine, obstructed labour and malpresentations of the fetus. Some studies have suggested surgical intervention for concerns of malignancy, tumor torsion. Tumor rupture, or obstruction principle of observation, finding that most ovarian masses can either remain uneventful or resolve throughout pregnancy and that the incidence of the above risks was actually low. Its most common cause in pregnancy is a corpus luteum cyst, which usually regresses spontaneously by the second trimester. Ovarian torsion, therefore, occurs most frequently in the first trimester, occasionally in the second, and rarely in the third.

\section{CONCLUSION}

Large intra-abodominal tumours change the anatomy, physiology, and psychology of the patient. The musculature of the abdominal wall becomes stretched beyond the limits of rapid recovery as the tumour increases in size, and the patient may be rendered immobile. Pregnancy, a fibroid uterus, ovarian cysts, hydatod cysts, hydronephrosis, lomphomas, and fluid collections may produce such swellings. Whatever the cause, the consequences of surgery are similar and attributable to the size of the tumour rather than to its distinctive pathology.

Any emergency surgery can be performed in any trimester depending on the type of urgency. Whenever possible surgeries should be considered in the second trimester as spontaneous abortion is less likely. General anesthesia is not an absolute contraindication but the combination of regional and general anesthesia has got better outcome. Regional anesthesia itself has better outcome results in pregnance patients undergoing non obstetric surgery, because of less multipharmacy needed, sooner mobility after surgery, and lesser drugs effect on fetus. Multidisciplinary approach in the peri-operative period involving anesthesiologist, obstetrician, surgeon, and neonatologist should be considered when applicable for a better coutcome

\section{REFFERRENCES}

[1] Shetti AN, Dhulkhed VK, Gujarati A, Swetha GS. Anesthetic Management of A Pregnant Patient with Pseudo-Pancreatic Cyst for Cysto-Gastrostomy. Case Report. Anesthesia: Essay and Researches. Takrouri MSM (Ed). Open Access. HTML format. Downloaded free from http://www.aeronline.org.on Tuesday Dec 30th, 2014.

[2] Aboulghar M, Mansour R, Serour G. Ovarian Cysts During Pregnancy: The Role of Ultrasonically Guided Transvaginal Aspiration. Ultrasound Obstet. Gynecol 2 (1992): 349-51

[3] Sinantyanta H, Sujana IBG. Management Anestesi pada Pasien dengan Kistoma Ovari Permagna. Laporan Kasus. Jurnal Anestesiologi Indonesia, 2013; 3: 225-31.

[4] Hoile RW. Hazards in The Management of Large Intra Abdominal Tumours-Aspects of Treatment-Annals of The Royal College of Surgeons of England, 1976; 58: 393-8.

[5] Elhalwagy H. Management of Ovarian Masses in Pregnancy. Medical Problems in Pregnancy-Trends in Urology Gynaecology and Sexual Health. January/ February, 2009. www.tugsh.com

[6] O'Hanlan KA. Case Report. Resection of A 303.2-Pound Ovarian Tumor. Gynaecologic Oncology, 1994; 54: 365-71.

[7] Hool A. Anaesthesia in Pregnancy for Non-Obstetric Surgery. Anaesthesia Tutorial of The Week 185. 28 June, $2010: 1$-10.

[8] Pelosi II MA, Pelosi III MA. A Novel Minilaparatomy Approach for Large Ovarian Cysts. Surgical Techniques. Obgmanagement. February 2004: 17-26.

[9] Kolluru V, Gurumurthy R, Vellanki V, Gururaj D. Torsion of Ovarian Cyst During Pregnancy: A Case Report. Biomed Central. Cases Journal 2009, 2 (9405): 1-3.

[10] Hoffman MS, Sayer RA. Adnexal Masses in Pregnancy. A Guide to Management Forego Surgery in Most Cases Until Delivery or Until The Risky First Trimester has Passed. OBG Management. March 2007: 27-34.

[11] Ovarian Cysts. Frequently Asked Questions. Womens Health gov. National Women's Health Information Center. U. S. Department of Health and Human Services. Office on Women's Health.

[12] Dilbraz S, Caliskan E, Dilbaz B, Aykan B, Sivaslioglu A, Haberal A. Laparoscopic and Transvaginal Ultrasound Guided Aspiration Cytology of Ovarian Cysts. Clinical Study. SSK Maternity and Womens Health Hospital Ankara, Turkey. Artemis, January 2003; 4 (1): 41-5.

[13] Ulker K, Ersoz M, Huseyinoglu U. Management of A Giant Ovarian Cysts by Keyless Abdominal Rope. Lifting Surgery (KARS). Turkey. Case Report. Kaflas J Med Sci 2011; 1 (1): 25-9.

[14] Kim SK, Kim JS, Park CH, Park JLV. A Case of Giant Ovarian Cyst Managed Successfully Through Laparoscopic Surgery. Case Report. Korean J Obstet Gynecol 2012; 55 (7): 534-7. 
[15] Shawki O, Soliman I, Ebrashy A, Sadek ME, Bahnassy A. Laparoscopic Management of Ovarian Dermoic Cysts. Middle East Fertility Society Journal, 2004; 9 (1): 58-64.

[16] Pre- and Post- Operative Instructions: Laparotomy (Ovarian Cystectomy, Oopharectomy, Myomectomy). Gynaecology Program. Mt Sinai Hospital. Joseph and Wolf Lebovic Health Complex.

[17] Milligan KR, Loughran PG, Moore JA, Weir P. Aortocaval Compression Secondary to An Ovarian Cyst. Case Report: 164-5.

[18] Doctor TP, Vakil SD. Anesthetic Management of Paraplegic Patient with Pregnancy for Spine Surgery (A Case Report). Indian J Anaesth 2003; 47 (2): 145-57.

[19] Van de Velden M, De Buck D. Anesthesia for Non Obstetric Surgery in The Pregnant Patient. Anesthesia.tk. Minerva Anesthesiologica, 2007; 73 (4):235-41.

[20] Buckle AER. The Infected Ovarian Cyst. Postgrad. Med. J, 1996; 42 : 30-7

Picture

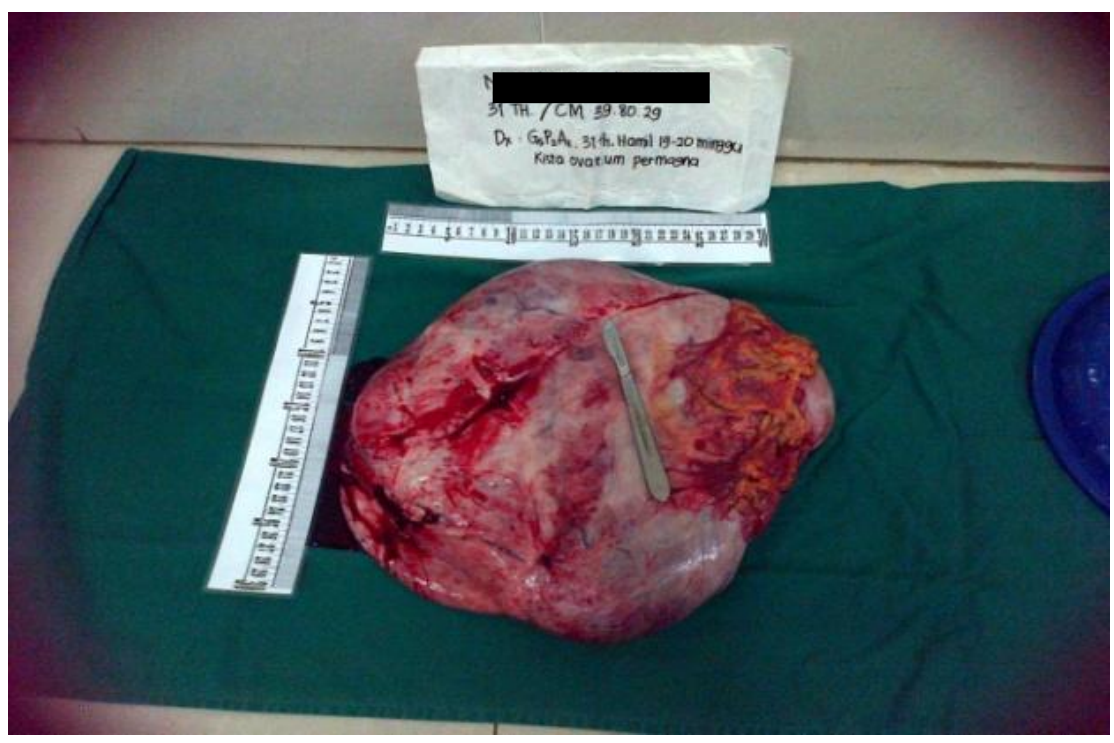

